\title{
VERBAL ANALYSIS OF THE INVESTMENT RISK IN CONSTRUCTION
}

\author{
Leonas Ustinovičius ${ }^{1}$, Dmitry Kochin ${ }^{2}$ \\ ${ }^{1}$ Vilnius Gediminas Technical University, Saulètekio al. 11, LT-2040 Vilnius, Lithuania \\ ${ }^{2}$ Institute for Systems Analysis, Prospect 60 let Octiabria, Moscow, Russia, \\ e-mail: 1eonasu@st.vtu.lt, ${ }^{29}$ dco@mail.ru \\ Received 11 April, 2003
}

\begin{abstract}
Obtaining of reliable enough estimations of investment quality is a complex task, since there is no universal indicator of capital recovery probability. Classification is a very important aspect in decision making. This means the prescription of projects to the particular classes. Very often it is stated that classes in decision making are determined by the particular parameters, i.e. the efficiency of technical and technological decisions, credit value determination of the project, etc. This article presents a verbal method of determining investment risk in construction. The problem under consideration is investment assessment depending on the risk level. Formally the problem is stated as multicriteria classification one. A hierarchical approach to considered effectiveness indicators is proposed. The proof of effectiveness of the method is presented. A procedure of method application is described for a practical task.
\end{abstract}

Keywords: investment risk in construction, decision-making, verbal analysis methods; methods of solving multicriteria classification problems, estimation of efficiency of investments

\section{Introduction}

Investment risk management is a common practice of any bank providing loans for projects. The evaluation of credit risk should be made at various phases of the project $[1,2]$. It may be stated that risk management implies that all the procedures should be rigorously followed at any phase of the project, the risk exposure depending on the output and accuracy.

Making a reliable qualitative analysis of the investments is a complicated problem [3], since the criterion for assessing the probability of capital recovery has not been found yet. There are many indicators (factors, criteria), which should be taken into account $[4,5]$. Each such criterion influences the capital recovery probability. Besides, credit classification for bank internal needs should take into account some other parameters of credit projects and debtor companies. The total credit quality is complex enough function of its distinct components. The circumstances, under which the bank works, are continuously changing because of general economic situation change.
That is why the rules of credit quality assessment can currently base only on a bank top management policy and an experience and an intuition of a bank leaders.

Ranking the investment projects according to risk exposure may be performed by the experienced bank loan operator or by aggregating the judgements perceived from bank experts. Both methods may be used in evaluating the investment projects.

Classification is a very important aspect in decision making. This means the prescription of projects to the particular classes. Very often it is stated that classes in decision making are determined by the particular parameters, i.e. the efficiency of technical and technological decisions, credit value determination of the project, etc. Classified projects are described by assessing various efficiency criteria that could be both qualitatively and quantitatively expressed.

In fact, many different methods for solving multicriteria classification problems are widely known. ORCLASS method, as an ordinary classification, was one of the first methods designed to solve these kinds of problems [6]. Then more recent methods appeared, 
such as DIFCLASS [7], CLARA [8] and CYCLE methods [9]. Verbal analysis approaches to the assessment and classification were applied to analyse and assess bank loans.

In multicriteria environment it is hardly possible to achieve this without resorting to special techniques. This article presents verbal method of determining investments risk in construction.

\section{Formal problem statement}

Given:

$\mathrm{G}$ - a feature, corresponding to specified purpose of the task (a value of investment project and so on).

$K=\left\{K_{1}, K_{2}, \ldots, K_{N}\right\}-$ a set of criteria, by which each alternative (investment project) is assessed.

$S_{q}=\left\{k_{1}^{q}, \ldots, k_{w_{q}}^{q}\right\}-$ for $\mathrm{q}=1, \ldots, \mathrm{N}-$ a set of estimations on criterion $\mathrm{K}_{\mathrm{q}}, \mathrm{w}_{\mathrm{q}}-\mathrm{a}$ number of grades on criterion $\mathrm{K}^{\mathrm{q}}$; estimations in $\mathrm{S}_{\mathrm{q}}$ are ordered by increasing typicalness for the feature $\mathrm{G}$;

$\mathrm{Y}=\mathrm{S}_{1} \times \ldots \times \mathrm{S}_{\mathrm{N}}-$ a state space of alternatives to be classified. Each alternative is described by a set of estimations by criteria $K_{1}, \ldots, K_{N}$ and can be presented as a vector estimation $y \in Y$, where $y=\left(y_{1}, y_{2}, \ldots, y_{N}\right), y_{q}$ is an index of estimation from $S_{q}$ set.

$C=\left\{C_{1}, \ldots, C_{M}\right\}-$ a set of decision classes, ordered by increasing degree of expression of feature $\mathrm{G}$.

A binary relation of strict dominance is introduced:

$$
P=\left\{\begin{array}{cl}
(x, y) \in Y \times Y \mid & \forall q=1 \ldots N \\
x_{q} \geq y_{q} & \exists q_{0}: x_{q_{0}}>y_{q_{0}}
\end{array}\right\}
$$

It is easy to note, that this relation is antireflexive, antisymmetric and transitive. It is useful to consider also reflexive, antisymmetric, transitive binary relation of weak dominance $Q$ :

$$
Q=\left\{(x, y) \in Y \times Y \mid \forall q=1 \ldots N, x_{q} \geq y_{q}\right\} .
$$

Goal: on the basis of DM preferences to create imaging $F: Y \rightarrow\left\{Y_{i}\right\}, i=1, \ldots, M$, where $Y_{i}$ - a set of vector estimations belonging to class $N_{i}$ ), satisfying the condition of consistency:

$$
\forall x, y \in Y: x \in Y_{i}, y \in Y_{j},(x, y) \in P \Rightarrow i \geq j .
$$

\section{Verbal decision analysis methods for classification of alternatives}

In this chapter some most frequently used verbal ordinal classification methods are considered. All these methods belong to Verbal Decision Analysis group and have the following common features [6]:

1. Attribute scale is based on verbal description not changed in the process of solution, when verbal evaluation is not converted into the numerical form or score.

2. Interactive procedure of classification is performed in steps, where the DM is offered an object of analysis (construction investment project). A project is presented as a short set of rankings. The DM is familiar with this type of description, therefore he/she can make the classification based on his/her expertise and intuition.

3. When the DM has decided to refer a project to a particular class, the decisions are ranked on the dominance basis. Classification of the project makes available the informaion about other classes of projects related with it by the relationship of dominance. Thus, indirect classification of all the projects can be made based on one DM decision.

4. A set of projects dominating over a considered project are referred to as domination cone. A great number of projects have been classified many times. This ensures error - free classification. If the DM makes an error, violating this principle, he/she is shown the conflicting decision on the screen and is prompted to adjust them.

5. In general, a comprehensive classification may be obtained for various numbers of the DM decisions and phases in an interactive operation. The efficiency of multicriteria classification technique is determined with account of the number of questions to DM needed to make the classification. This approach is justified, because it takes into consideration the cost of the DM's time and the need for minimizing classification expenses.

Let us review several most used methods in more detail.

Method ORCLASS [6]. This method (Ordinal CLASSification) allows to build classification subsequently, to check the information for consistency and to get general decision rules. The method uses a notion of the most informative alternative, classification of which allows to implicitly assign to the classes maximum number of other alternatives. ORCLASS takes into account possibilities and limitations of human information processing system. 
Method assessment: The disadvantage of the method is low effectiveness due to great number of questions to DM for building full classification.

Method CLARA [8]. This method (CLAssification of Real Alternatives) is based on ORCLASS, but is designed to classify not full set of alternatives (Y space), but some given subset. Another common application of CLARA is classification of full set with large number of exclusions, i.e. alternatives with impossible combinations of estimations. In both cases CLARA demonstrate high effectiveness.

Method DIFCLASS [7]. This method was the first to use dynamic construction of chains covering Y space for selection of questions to DM. However, area of application for DIFCLASS is bounded to tasks with binary criteria scales and two decision classes.

Method CYCLE [9, 10]. CYCLE (Chain Interactive Classification) algorithm overcomes DIFCLASS restrictions, generalizing the idea of dynamic chain construction to the area of ordinal classification task with arbitrary criteria scales and any number of decision classes. The chain here means ordered sequence of vectors $\left\langle x_{1}, \ldots, x_{d}\right\rangle$, where $\left(x_{i+1}, x_{i}\right) \in P$ and vectors $x_{i+1}$ and $x_{i}$ differs by one by one of the components.

Method assessment: As comparisons demonstrate, the idea of dynamic chain construction allows to get an algorithm close to optimal by minimum number of questions to DM necessary to build full classification. An experience of ordinal classification system application demonstrated that problem formalization, introduction of classes and criteria structure allowed solving the classification problem using high effective methods.

The method can be successfully applied to classification of investment projects after definite revision of decision classes and criteria used.

\section{Verbal analysis of the investment risk}

Classification of projects is one of the multiple criteria problems within the framework of decision making system [11-14]. Such problems can be solved by CYCLE technique developed at the Institute of System Analysis of the Russian Academy of Sciences [9, 15]. The latter technique allows the classification to be developed in a series of successive steps, checking the conflicting information and arriving at a general method of solution. The method described takes into account the possibilities and limitations of the human data processing system [16].

\section{Method CYCLE}

Let us consider metric $\rho(x, y)$ in discrete space $Y$ defined as:

$$
\rho(x, y)=\sum_{q=1}^{N}\left|x_{q}-y_{q}\right|
$$

Let us define index of vector $y \in Y$ (denoted as $\|y\|)$ as $\rho(\overrightarrow{0}, y)$, that is the sum of its components. For vectors $y, y \in Y$ таких, что $(x, y) \in P$, рассмотрим множество

$$
\Lambda(x, y)=\{v \in Y \mid(x, v) \in Q(v, y) \in Q\},
$$

that is a set of vectors weakly dominating $y$ and weakly dominated by $x$. Having denoted $y^{\prime}=(1, \ldots$, $1), y^{\prime \prime}=\left(w_{1}, \ldots, w_{N}\right)$, it is easy to see that $\Lambda\left(y^{\prime \prime}, y^{\prime}\right)$ coincide with the entire space $Y$. We also introduce a set

$$
L(x, y)=\left\{v \in \Lambda(x, y)\|v\|=\frac{\|x\|+\|y\|}{2}\right\},
$$

that is vectors from $\Lambda(x, y)$ set equidistant from $x$ and $y$ (here and from now on dividing is done without a reminder). We will need numerical functions $C^{U}(x)$ and $C^{L}(x)$ defined on $Y$, which equal correspondingly to maximum and minimum number of class allowed for $x$, that is a class for $x$ not violating condition of consistency (2). Let us consider vector $x$ classified and belonging to class $C_{k}$ if for $x$ the following condition is true:

$$
C^{U}(x)=C^{L}(x)=k
$$

Let us define procedure $S(x)$ (spreading by dominance). It is assumed that classification of $\mathrm{x}$ is known: $x \in Y_{k}$ (that is $C^{U}(x)=C^{L}(x)=k$ ). Therefore for all $y \in Y$ such as $(x, y) \in P$ and $C^{U}(y)>k$ function $C^{U}(y)$ is redefined so $C^{U}(y)=k$. Similarly for all $\mathrm{z} \in \mathrm{Y}$ such as $(z, x) \in P$ and $C^{L}(z)<k$ function $C^{L}(z)$ is redefined so $C^{L}(z)=\mathrm{k}$.

\section{Basic mechanism of the CYCLE algorithm.}

Let us denote $D(a, b)$ - procedure of classification on $\Lambda(a, b)$ set using the idea of dynamic construction of chains linking vectors $a$ and $b$. It is assumned that ( $a$, $b) \in P$, classes of vectors $a$ and $b$ are known: $a \in Y_{k}, b \in Y_{l}$.

The algorithm is as follows:

1. For each vector $x \in L(a, b)$ the steps $2-4$ are done.

2. If a class for $y$ is unknown $\left(C^{L}(x)<C^{U}(x)\right)$ then 
$y$ is presented to DM for classification. Suppose, $x \in Y_{r}$. The spreading by dominance $S(x)$ is being done.

The condition of consistency is being checked (2).

3. If $r<k$ and $(a, x) \in P$ then perform $D(a, x)$.

4. If $r<k$ and $(a, b) \in P$ then perform $D(x, b)$.

During classification of vector $\mathrm{x}$ on the second step a DM can make a mistake, and a pair of vectors $x, y \in Y$ violating the consistency condition (2) appears. Procedure $R$ of resolving contradictions consists in following. Let us denote the set of vectors explicitly classified by DM as $E$. So while $E$ contains a pair of vectors violating (2) such a pair is presented to DM with a proposition to change a class for one or two vectors. After that functions $C^{U}$ and $C^{L}$ are redefined to their initial values and spreading by dominance $S(v)$ is done for each $v \in E$.

Generally speaking, parameters of the algorithm including number of questions to DM depend on the choice of vector $x$ on the first step. The following heuristics is proposed: among all not yet classified vectors from $L(a, b)$ set one chooses the object, which explicitly dominates maximum number of unclassified vectors. That is, one chooses vector

$$
\begin{gathered}
x^{*}=\arg \max _{x \in L(a, b)} \mid\{y \in Y \mid(x, y) \in P \text { or } \\
\left.(y, x) \in P, \rho(x, y)=1, C^{L}(y)<C^{U}(y)\right\} \mid .
\end{gathered}
$$

At the very high level CYCLE algorithm looks as following:

1. For each $\mathrm{v} \in \mathrm{Y}$ possible classes are set to $C^{L}(y)$ $=C^{U}(y)=\mathrm{M}$.

2. DM is presented vectors $y^{\prime}$ and $y^{\prime \prime}$, spreading by dominance $S\left(y^{\prime}\right)$ and $S\left(y^{\prime \prime}\right)$ are done.

3 . If classes for $y^{\prime}$ and $y^{\prime \prime}$ differ then procedure $D\left(y^{\prime \prime}, y^{\prime}\right)$ is performed.

Algorithm features: Statement 1. At the end of CYCLE algorithm the space $Y$ will be fully classified, that is $\forall y \in Y \quad C^{L}(y)=C^{L}(y)$.

Proof. Let us consider a chain in the form $\mathfrak{R}^{0}=\left\langle y^{\prime}, \ldots, y, \ldots, y^{\prime \prime}\right\rangle$. Under $C(x)$ we understand a class for vector $y$. Vectors $y$ and $y^{\prime \prime}$ are classified by DM at the second step of CYCLE algorithm. We make an assumption, that $C\left(y^{\prime}\right)<C\left(y^{\prime \prime}\right)$, because if $C\left(y^{\prime}\right)>C\left(y^{\prime \prime}\right)$ then the condition of consistency (2) is violated, and if $C\left(y^{\prime}\right)=C\left(y^{\prime \prime}\right)$ then classification is trivial.

Performing procedure $D\left(y^{\prime \prime}, y^{\prime}\right)$ on the first step a computer chooses, and then present to DM for classification some vector $x^{0}=\mathfrak{R}^{0} \cap L\left(y^{\prime \prime}, y^{\prime}\right)$. This intersection consists of only one vector due to Lemma 1, which will be proved later. To fix the idea let us assume that $\left\|y^{\prime}\right\|<\left\|x^{0}\right\| \leq\|y\|<\left\|y^{\prime \prime}\right\|$, since the case $\|y\| \leq\left\|x^{0}\right\|$ is considered analogously. From condition of consistency (2) one can conclude, that $C\left(x^{0}\right) \leq C\left(y^{\prime \prime}\right)$. There are two cases:

$C\left(x_{0}\right)=C\left(y^{\prime \prime}\right)$, in this case all vectors on the part of the chain $\Re^{0}$ between $x^{0}$ and $y^{\prime \prime}$ are assigned to the same class, consequently, vector $y$ is also classified;

$C\left(x_{0}\right)<C\left(y^{\prime \prime}\right)$, let us consider chain $\Re^{1}=\left\langle x^{0}, \ldots, y, \ldots, y^{\prime \prime}\right\rangle$ and repeat for it the same speculations as for chain $\Re^{0}$, namely - a procedure $D\left(y^{\prime \prime}\right.$, $\left.y^{0}\right)$ is performed, a computer chooses and present for a classification vector $x^{1}=\Re^{1} \cap L\left(y^{\prime \prime}, y^{\prime}\right)$ and so on.

Thus, subsequently building chains $\mathfrak{R}^{0}, \mathfrak{R}^{1}, \ldots$, containing $y$ we either find fully classified part of the chain (as in first case), or get to chain $\Re^{1}$ of length 2 , since it is easy to see, that $\left|\Re^{i+1}\right|=1 / 2\left|\Re^{i}\right|+1$.

But the algorithm ensures that before starting procedure $D(v, u)$ both vectors $v$ and $u$ are already classified, and therefore vector $y$ will also be classified.

Lemma 1. For any $x, y \in Y$ such as $(x, y) \in P$ and for any chain $\Re=\langle x, \ldots, y\rangle$, cardinality of the set $\Re \cap L(y, x)$ equals to 1 .

Proof. Since for any vector $v \in \Re$ the condition $(v, x)$ $\in Q$ and $(y, v) \in Q$ is true then $\Re \subseteq \Lambda(y, x)$. According to the definition, a chain contain exactly one vector with every value of index in interval $\|y\| . . .\|x\|$, and therefore $\frac{\|x\|+\|y\|}{2}$.

Statement 2. A classification build with the help of CYCLE algorithm is consistent that is a condition of consistency (2) is satisfied.

Proof. Assume there are some vectors $y^{*}, x^{*} \in Y$ such as they violate consistency condition (2):

$$
x^{*}, y^{*} \in P, x^{*} \in Y_{k}, y^{*} \in Y_{l}, k<l .
$$

Vector $y^{*}$ was either explicitly classified by DM $\left(y^{*}\right.$ $\in E$ ), or there are $x^{1}, x^{2} \in E$ such as $x^{1} P x^{*} P x^{2}$ and $y^{1}, y^{2} \in Y_{k}$. In first case it is considered that $x^{1}=x^{2}=$ $x^{*}$. Analogously, either $x^{*} \in E$, or there are $x^{1}, x^{2} \in E$ such as $y^{\prime} P y^{*} P y^{2}$ and $y^{1}, y^{2} \in Y_{l}$. However, procedure $R$ guarantees satisfaction of (2) for $E$ set, that is in particular from $\left(x^{1}, x^{2}\right) \in P$ due to transitivity of $P$ (since: $x^{1} P x^{*} P y^{*} P y^{2}$ ), $x^{1} \in Y_{k}, y^{2} \in Y_{l}$ ) we can conclude that $k \geq l$, and this contradicts with (3). Thus, the condition of consistency (2) is satisfied for entire space $Y$. 


\section{Risk classification and description of main criteria used}

After a series of iterations, carried out under methodological control of consultants from ISA RAS the following final decision classes were chosen (Fig):

1a. The highest quality (Hi class): the investor meets all the requirements, being completely reliable, a credit line is available to the investor, the limit of crediting is defined.

1b.High quality: a comprehensive analysis of the company's performance and the investment project has shown high probability of the investor's satisfying all the requirements of the contract.

2a. Sufficient quality: the investor may be faced with minor problems in meeting the requirements of the contract.

2b.Low quality: the investor may be faced with more serious problems in meeting the requirements of the contract.

3. Poor quality: the investor has problems with paying loan interest though able to cover the loan.

4. Losses: the investor is unable to cover the debt.

At the lowest hierarchical level, 6 groups of efficiency criteria were formed: debt service according to the investment project; evaluation of the investment project; the importance of the investor to the bank; reliability of investor; evaluation of the financial state of investor; stability and potential of the investing company. More detailed description of the above groups is given below.

1. The group "Debt service according to the investment project" includes: evaluation of debt service; liquidity; prediction of debt service cost; sufficiency of debt service.

2. The group "The evaluation of the investment project" includes the criteria of probability and the conditions of making preliminary changes determining the quality of the project analysis.

3. The importance of the investor to the bank is a separate criterion.

4. The group "Reliability of investor" includes: state of investor; evaluation of the behaviour of the investor's representative in negotiations; crediting history; some evidence proving the absence of compromising information about investor.

5. The group "Financial state of investor" includes: current accounts and current assets in the bank; type of financial stability; debts to other banks.

6. The group "Stability and potential of the investing company" includes: general level of company's management; availability of long - term objectives and plans to achieve them; stability of the company in the context of other related factors (i.e. economic situation, market changes) in the period of crediting.

The above groups of 6 attributes can be easily transformed into some more general groups ( 1 and 3, 3 and 4, 5 and 6) under the following headings:

- substantiation of crediting;

- evaluation of debtor as an enterprise;

- financial state and the potential of debtor.

It is now necessary to classify by risk all possible investment projects by their multicriteria description. The quality of results obtained should be validated thoroughly.

Initially a classification is built on the second layer inside of the efficiency criteria group. The role of classes is played by general marks of first layer of hierarchy. After classification these general marks are filled with concrete sense. Then a classification on the first level of hierarchy is built. As a result we get decision rules for determining the risk level for any investment project.

DM can tell the risk of investment project on the basis of available information. One should note, that it is possible $t$ use criteria of first hierarchy level only. Having difficulties in assessment DM can use more detailed second layer. The possibility to use second hierarchy level exists even for particular first layer criterion.

\section{Conclusions}

The risk of construction investment project was defined by a verbal analysis based on the classification decision support method CYCLE, allowing the risk evaluation according to the specified classes by the suggested criteria of determining risk exposure level.

As comparisons demonstrate, the idea of dynamic chain construction allows to get an algorithm close to optimal by minimum number of questions to DM necessary to build full classification.

The developed method was validated by solving actual problems of selecting the best variants of construction and reconstruction investment projects. The investment projects selected were implemented. 


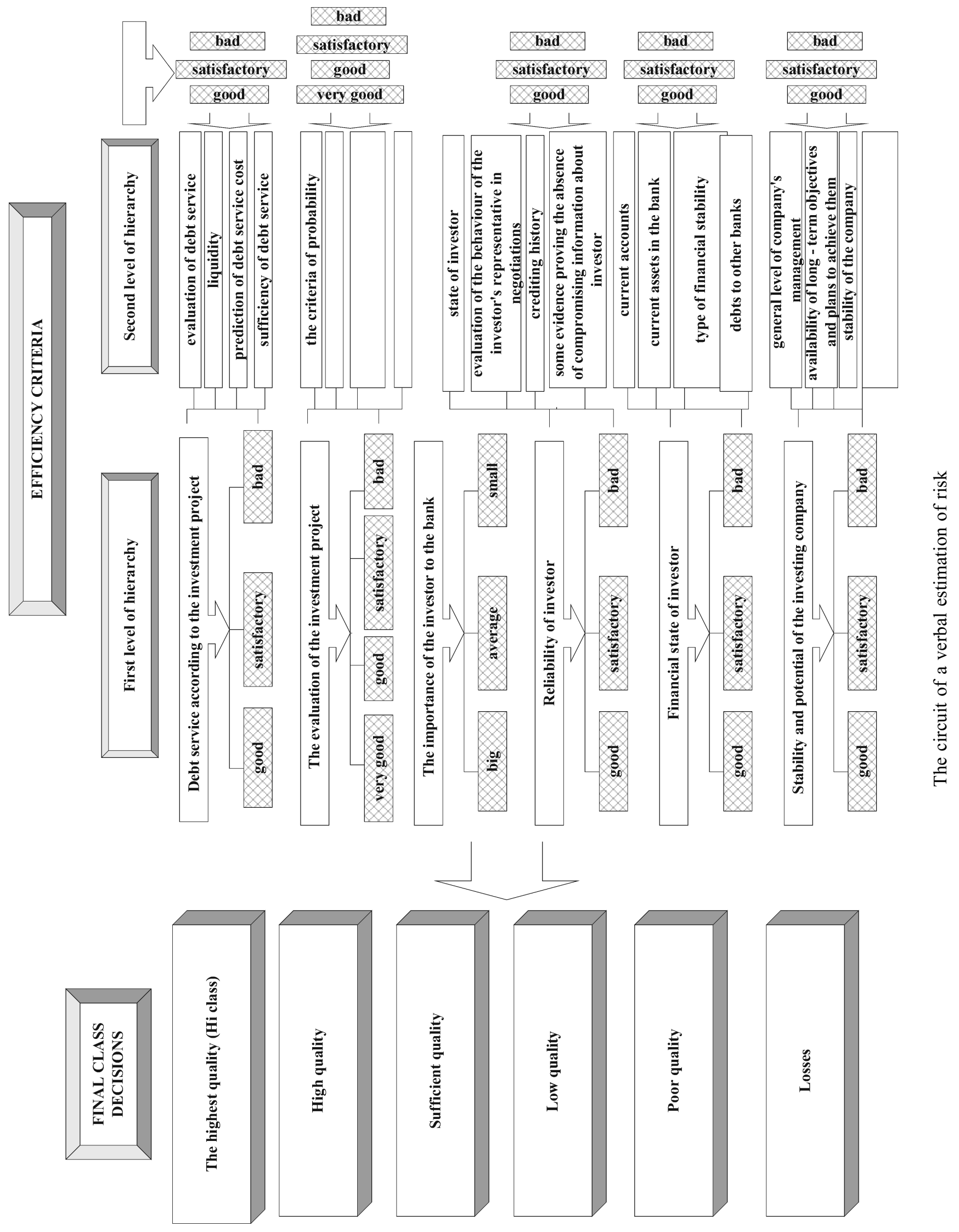




\section{References}

1. Slowinski R., Zopounidis C. Application of the rough set approach to evaluation of bankruptcy risk. International Journal of Intelligent Systems in Accounting Finance and Management, Vol. 4, No. 1, 1995, p. 27-41.

2. Greco S., Matarazzo B., Slowinski R. A new rough set approach to evaluation of bankruptcy risk. In: Zopounidis, C. (Ed.), Operational Tools in the Management of Financial Risks, Kluwer Academic Publishers, Dordrecht, 1998, p. 121-136.

3. Ustinovičius L., Jakučionis S. Multi- criteria analysis of the variants of the old town building renovation in the marketing. Statyba (Civil Engineering), Vol. 6, No. 6, 2000, p. 212-222.

4. Zavadskas E. K., Peldschus F., Ustinovichius L. Development of software for multiple criteria evaluation. Informatica, Vol. 14, No. 2, 2003, p. 259-272.

5. Larichev O., Kochin D., Ustinovichius L. Multicriteria method of choosing the best alternative for investments. International Journal of Strategic Property Management, Vol. 7, No. 1, 2003, p. 33-43.

6. Larichev O., Mechitov A., Moshovich E., Furems E. Revealing of expert knowledge, Moscow: Publishing house Nauka, 1989 (in Russian).

7. Larichev O., Bolotov A. System DIFKLASS: construction of full and consistent bases of expert knowledge in problems of differential classification. The scientific and technical information, a series 2. Information processes and systems (Информационные процессы и системы), No. 9, 1996 (in Russian).

8. Larichev O. I., Kochin D. Yu. and Kortnev A. V. Decision support system for classification of a finite set of multicriteria alternatives. Decision Support Systems, Vol. 33, No. 1, 2002, p. 13-21.
9. Aksanov A., Borisenkov P., Larichev O., Nariznij E., Rozejnzon G. Method of multicriteria classification CYCLE and its application for the analysis of credit risk. Economy and mathematical methods (Экономика и математические методы), Vol. 37, No. 2, 2001, p. 1421. (in Russian).

10. Larichev O., Leonov A. Method CYCLE of serial classification of multicriteria alternatives. The report of the Russian Academy of sciences, 2000, December (in Russian).

11. Larichev O., Moshkovich H. An Approach to Ordinal Classification Problems. International Transaction in Operational Research, Vol. 1, No. 4, 1994, p. 375-386.

12. Larichev O., Moshkovich H. Qualitative methods of decision making. Moscow.: Nauka, Fizmatlit, 1996 (in Russian).

13. de Montgolfier J., Bertier P. Approche multicritere des problemes de decision. Paris: Editions Hommes et Techniques, 1978 (in French).

14. Greco S., Matarazzo B., Slowinski R. Rough sets methodology for sorting problems in presence of multiple attributes and criteria. European Journal of Operational Research, Vol. 138, No 2, 2002, p. 247-259.

15. Larichev O., Borisenkov P., Asanov A., Nariznij G., Rozejnzon G. The Development and introducing the automaticed system of estimation of quality of credits in commercial bank. Audit and the financial analysis (Аудит и финансовый анализ), №. 1, 2001, p. 196200 (in Russian).

16. Solso P. Cognitive psychology. Moscow: Trivola (in Russian). 